\title{
林木根呼吸及测定方法进展
}

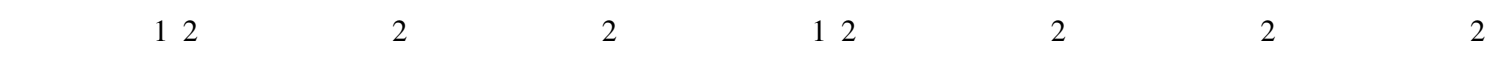

（1 福建师范大学地理科学学院, 福州 350007)（2 福建农林大学林学院, 福建南平 353001)

摘 要 森林土壤呼吸的近 $2 / 3$ 是由林木根呼吸产生的 林木根呼吸对估计森林 $\mathrm{C}$ 吸存及构建森林生态系统碳动 态模型有重要意义, 是全球碳循环研究的一个重要组成部分。林木根呼吸包括生长呼吸和维持呼吸, 不同森林生 态系统林木根呼吸对土壤呼吸的贡献大多在 $40 \%$ ～60\%范围内 林木根呼吸在生长季节较高而休眠季节较低。测 定林木根呼吸的主要方法有排除根法、离体根法、同位素法和原位 PVC 管气室法, 前两者相对简单、成本低, 常用于 森林生态系统中; 同位素法可原位测定根呼吸, 对土壤干扰较小, 但不易操作, 且成本高。根呼吸受土壤温度、根直 径大小、根组织 $\mathrm{N}$ 浓度、环境 $\mathrm{CO}_{2}$ 浓度、土壤湿度、养分有效性等因素的影响。今后的研究应集中在以下方面:1)探 讨和比较不同条件下测定根呼吸组成(生长呼吸、维持呼吸)的最合适方法 ,2)加大在野外条件下使用有效方法分 离根呼吸和根际微生物呼吸的力度 3) 对森林生态系统根呼吸动态进行长期的定位研究; ;)进一步加强研究不同 气候带, 不同森林类型林木根呼吸, 并将研究尺度从气室扩大到区域或全球水平 ;5)加强林木根呼吸对全球变化的 响应及机制的研究; 6) 对林木根呼吸进行多学科合作研究将为全球 C 循环做出新的贡献。

关键词 林木根呼吸 影响因素 测定方法 全球变化

\section{A REVIEW OF TREE ROOT RESPIRATION : SIGNIFICANCE AND METHODOLOGIES}

\author{
YANG Yu-Sheng $^{12}$ DONG Bin ${ }^{2}$ XIE Jin-Sheng ${ }^{2}$ CHEN Guang-Shui ${ }^{12}$ \\ LI Ling ${ }^{2}$ LIU Dong-Xia ${ }^{2}$ and LI Zhen ${ }^{2}$ \\ (1 College of Geography Science , Fujian Normal University, Fuzhou 350007 , China) \\ (2 College of Forestry, Fujian Agriculture and Forestry University , Nanping 353001 , China)
}

\begin{abstract}
Tree root respiration is a major contributor to soil $\mathrm{CO}_{2}$ pools , and 2/3 of total forest soil respiration is from root respiration. Information about root respiration is important for understanding the implications of environmental change on soil carbon cycling and carbon sequestration , and for the development of carbon models of forest ecosystem dynamics. Furthermore, the global $\mathrm{CO}_{2}$ flux from root respiration is estimated to be about $18 \mathrm{Pg} \mathrm{G} \mathrm{a}{ }^{-1}$, which is an order of magnitude larger than that produced by anthropogenic sources of $\mathrm{CO}_{2}$. Therefore, changes in forest tree root respiration could have a significant impact on the future global carbon balance. Ecological research on tree root respiration is relatively recent and still very little research on this topic has been conducted in China. In this paper, We review the characteristics, methodologies and factors affecting tree root respiration for the purpose of stimulating new, domestic research on this topic.

Tree root respiration is composed of maintenance respiration and growth respiration. Maintenance respiration , the dominant component of total of total root respiration, is used to maintain the living biomass, and growth respiration, which is used to construct new biomass, is proportional to the amount of new dry matter synthesized. Root respiration rates vary significantly among forest types. The proportion of the total soil carbon flux that is attributable to live root respiration appears to be very high in cold, northern biomes , ranging from $50 \%$ to $93 \%$ in the arctic tundra and from $62 \%$ to $89 \%$ in boreal forests. In temperate zones, estimated proportions of the total soil respiration flux that is derived from live root respiration range from $33 \%$ to $50 \%$ in broad-leaved forests and from $35 \%$ to $62 \%$ in pine forests. Root respiration typically is $40 \%-60 \%$ of total soil respiration in most forests. Tree root respiration has significant seasonal dynamics with respiration greatest during the growing season and lowest during the dormant periods of the year.

Methods used to measure tree root respiration include root exclusion methods, in vitro root techniques , stable or radioactive isotope methods and in situ cuvette methods. Each approach has advantages and disadvantages. The first two methods are relatively simple and inexpensive and are commonly used in forest ecosystems. Isotope based methods provide quantitative answers with the least amount of disturbance to the soil and roots ,
\end{abstract}


but the complexity of the experimental setup and the high costs associated with the analysis of radioactive or stable $\mathrm{C}$ isotopes are major disadvantages. The in situ cuvette method is considered an important method for future studies. Critical factors influencing rates of tree root respiration include soil temperature, root diameter size , ambient $\mathrm{CO}_{2}$ concentration , soil moisture, and nutrient availability .

Despite intensive research in recent years , many uncertainties remain in this dynamic and important field of research. Topics of particular importance include :1) Discussion and comparison of the appropriate methods for accurately measuring tree root respiration ;2) Application of effective methods for separating root respiration and rhizosphere respiration in the field ;3) Long-term research on the dynamics of tree root respiration in forest ecosystems ;4) Studies of tree root respiration in different ecosystems and climatic zones ;5) How to scale up from small chambers to the stand level, ecosystem level , regional or global level ;6) Understanding the mechanistic response of tree root respiration to global climate change ; and ,7) Inter-disciplinary research on tree root respiration to understand its role in the global carbon cycle.

Key words Tree root respiration, Methodologies , Global climate change , C cycle

林木根呼吸每年所消耗的呼吸底物占林木总光 合作用产物的 50\%左右 (Lambers et al , 1996), 是森 林生态系统 $\mathrm{C}$ 循环的重要 $\mathrm{C}$ 途径之一，其动态变化 将对森林生态系统乃至全球 $\mathrm{C}$ 平衡产生深远影响。 林木根呼吸亦是森林地下 $\mathrm{C}$ 库的重要通量之一(可 占森林土壤呼吸的 $10 \% \sim 90 \%$ ), 在森林地下 $\mathrm{C}$ 库 $\mathrm{C}$ 预算及确定森林地下 $\mathrm{C}$ 库是 $\mathrm{C}$ 源或汇上起着关键 性作用 (Hanson et al.,2000)。另外, 有关林木根呼 吸的数据亦是构建和完善各种尺度森林生态系统碳 动态模型所缺乏的和所必需的 (Hogg ,1999)。由于 其在森林生态系统 $\mathrm{C}$ 平衡和 $\mathrm{C}$ 预算中的重要性, 林 木根呼吸研究在 1995 年的树木根系生理动态过程 国际会议上被列为优先研究的领域。此后, 有关林 木根呼吸的报道明显增加 (Widén \& Majdi，2001; Desrochers et al . , 2002 ; Burton et al . , 2002 ;Burton \& Pregitzer ,2003) , 而我国有关林木根呼吸的研究几乎 是空白(上官周平等，2000)。鉴于此，本文回顾了目 前国际上林木根呼吸研究动态和相关测定方法, 希 望为推动我国相关领域的研究提供参考。

\section{1 林木根呼吸特性}

林木根呼吸是森林土壤呼吸中的自养呼吸 ( $\mathrm{Au}-$ totrophic respiration)部分，包括细根呼吸和粗根呼吸。 早期研究中根呼吸由活根组织呼吸、共生菌根呼吸 和参与分解根分泌物及根际中刚死亡根组织的微生 物的呼吸, 实际上包括了根呼吸 (自养呼吸)和根际 微生物呼吸(异养呼吸) (Toland \& Zak ,1994 ;Thierron \& Laudelout ,1996 ; Bouma \& Bryla , 2000)。而随着研 究的进展, 精确区分根呼吸和根际微生物呼吸则已 成为准确估算森林生态系统 $\mathrm{C}$ 平衡的需要 (Hanson et al. ,2000 ; Widén \& Majdi , 2001 ; Irvine \& Law , 2002 ; Burton \& Pregitzer ,2003)。Amthor (2000) 把根
呼吸分为维持现有组织的维持呼吸 (Maintenance respiration)和用于构建新组织的生长呼吸 (Growth respiration)。维持呼吸与林木活原生质组成和数量有 关，一般用休眠季节测定的根呼吸值来表示 (Vose \& Ryan，2002）;而生长呼吸则与新合成的干物质数量 成比例 (Amthor，2000)。

根系呼吸占土壤呼吸的 $10 \% \sim 90 \%$,与植被类 型、测定季节和方法有关 (表 1)。根呼吸占土壤呼 吸的比例在北方生物群落中较高, 其中北极冻原为 50\% 93\% (Raich \& Tufekcioglu ,2000) 、北方森林 为 $62 \% \sim 89 \%$ (Bonan, 1993 ; Ryan et al , 1997) ;在 温带 根呼吸占土壤呼吸的比例阔叶林为 $33 \%$ 50\%（Bowden et al. 1993 ; Nakane et al. , 1996)，松 树林为 35\% 62\% (Ewel et al., 1987 ; Striegl \& Wickland，1998）。而世界范围内根呼吸占土壤呼吸 的比例主要集中在 40\% 60\% (Raich \& Schlesinger, 1992 ;Bowden et al . 1993; Epron et al . 1999 ;Raich \& Schlesinger ,2000 ; Widén \& Majdi ,2001 ; Irvine \& Law , 2002)。

林木根呼吸有明显的季节变化，一般生长季节 较高, 休眠季节较低 ( Toland \& Zak, $1994 ;$ Zogg et al . , 1996 ; Maier \& Kress ,2000 ; Casals et al ., 2000 ; Coleman et al . ,2002) ;而根呼吸的昼夜变化有些学 者认为较明显 (Morén \& Lindroth，2000), 有些则否 (Widén \& Majdi，2001)。根呼吸时空变化方面的信 息对精确估计根呼吸对森林碳平衡的贡献非常有用 (Stoyan et al . 2000)。

Ebert 和 Lenz (1991) 发现苹果 (Malus pumila) 根 呼吸在 8 月最高, 2 月最低; 傍晚根呼吸大于黎明 的。Desrochers 等 (2002) 发现生长季节的细根呼吸 速率几乎是休眠季节的两倍。Widén 和 Majdi (2001) 发现欧洲赤松-云杉( Pinus sylvestris/Picea abies) 混交 
表 1 不同森林类型和测定方法下根/根际对土壤呼吸的贡献

Table 1 Estimates of percent root/rhizosphere contributions to total soil respiration ( $\mathrm{RC}$ ) by forest type and experimental approach

\begin{tabular}{|c|c|c|c|}
\hline $\begin{array}{l}\text { 物种 } \\
\text { Species }\end{array}$ & $\begin{array}{c}\text { 方法 } \\
\text { Approach }\end{array}$ & $\begin{array}{l}\text { 根/根际呼吸的贡献 }(\%) \\
\text { Root/rhizosphere contributions }\end{array}$ & $\begin{array}{l}\text { 参考文献 } \\
\text { Reference }\end{array}$ \\
\hline 水青冈 Fagus & 排除根法(林隙) (Rexcl. (Gap)) & 40 & Brumme, 1995 \\
\hline 水青冈/云杉 Fagus/Picea & 同位素- ${ }^{14} \mathrm{C}\left(\mathrm{I}-{ }^{14} \mathrm{C}\right)$ & 75 (Summer) ,25 (Winter) & Dörr \& Münnich , 1986 \\
\hline 山毛榉 Nothofagus & 排除根法 (Rexcl. ) & 60 & Epron et al. , 1999 \\
\hline 栎树 Quercus & 排除根法 (Rexcl. ) & 90 & Thierron \& Laudelout ,1996 \\
\hline 栋树 Quercus & 排除根法 (Rexcl.) & 52 & Kelting et al. , 1998 \\
\hline 栎树 Quercus & 排除根法(Rexcl.) & 23.3 & Rey et al. 2002 \\
\hline 栋/棫 Quercus/Acer & 排除根法 (Rexcl. ) & 33 & Bowden et al . , 1993 \\
\hline 糖槭 Acer saccharum & 排除根法 (Rexcl. ) & 60 & Pregitzer et al. 1998 \\
\hline $\begin{array}{l}\text { 欧洲赤松/云杉混交林 } \\
\text { Pinus sylvestris/Picea abies }\end{array}$ & 离体根法 (In vitro root methods) & $\begin{array}{l}33 \sim 62 \text { (May) } \\
12 \sim 16 \text { (October) }\end{array}$ & Widén \& Majdi , 2001 \\
\hline 湿地松 Pinus elliottii & 排除根法 (Rexcl. ) & 51 (9-year-old) & Ewel et al. , 1987 \\
\hline 湿地松 Pinus elliottii & 排除根法 (Rexcl. ) & 62 (29-year-old) & Ewel et al. , 1987 \\
\hline 火炬松 Pinus taeda & 排除根法 (Rexcl.) & 67 (December) & Edwards , 1991 \\
\hline 火炬松 Pinus taeda & 同位素- ${ }^{13} \mathrm{C}\left(\mathrm{I}-{ }^{13} \mathrm{C}\right)$ & 49 & Andrews et al. 1997 \\
\hline 火炬松 Pinus taeda & 同位素-13 C $\left(\mathrm{I}-{ }^{13} \mathrm{C}\right)$ & 55 & Andrews et al. 1999 \\
\hline 火炬松 Pinus taeda & 同位素- ${ }^{13} \mathrm{C}\left(\mathrm{I}-{ }^{13} \mathrm{C}\right)$ & 55 & Ellsworth et al. 1999 \\
\hline 火炬松 Pinus taeda & 排除根法 (Rexcl.) & $50 \sim 73$ & Maier \& Kress 2000 \\
\hline 脂松 Pinus resinosa & 排除根法 (Rexcl. ) & $40 \sim 65$ & Haynes \& Gower ,1995 \\
\hline 西黄松 Pinus ponderosa & 离体根法 (In vitro root methods) & 53 & Irvine \& Law , 2002 \\
\hline 北美短叶松 Pinus banksiana & 排除根法 (Rexcl. ) & 35 & Striegl \& Wickland ,1997 \\
\hline 欧洲赤松 Pinus sylvestris & 环割实验法 ( Girdling experiment) & 54 & Högberg et al. 2001 \\
\hline 黄杉 Pseudotsuga (1 年生 1-year) & 同位素 ${ }^{13} \mathrm{C} /{ }^{18} \mathrm{O}\left(\mathrm{I}-{ }^{13} \mathrm{C} /{ }^{18} \mathrm{O}\right)$ & 28 (April) & Lin \& Ehleringer , 1997 \\
\hline 日本柳杉 Cryptomeria japonica & 排除根法 (Rexcl. ) & $49 \sim 57$ & Ohashi et al. , 2000 \\
\hline 阔叶林 Broad-leaved forest & 排除根法(Rexcl.) & 51 & Nakane et al. ,1996 \\
\hline 阔叶林 Hardwood & 排除根法 (Rexcl. ) & $13 \sim 17$ & Catricala et al ., 1997 \\
\hline 温带阔叶林 Temperate broad-leaved forest & 排除根法(Rexcl.) & $70 \sim 80$ & Bowden et al. ,1993 \\
\hline 格氏栲 Castanopsis kawakamii & 排除根法(Rexcl.) & 23 (January) 57 (October) & 杨玉盛等1) \\
\hline 杉木 Cunninghamia lanceolata & 排除根法 (Rexcl. ) & 10 (January) 34( October) & \\
\hline
\end{tabular}

Rexcl. : 同位素标记法(标明同位素 , 如 ${ }^{14} \mathrm{C},{ }^{13} \mathrm{C}$ ) Root exclusion methods I-xxx are isotopiclabeling approaches ( with indicated isotope $\left(\mathrm{i} . \mathrm{e} .{ }^{14} \mathrm{C},{ }^{13} \mathrm{C}\right)$ ) 1) 杨玉盛等. 2002. 格氏栲天然林与人工林土壤呼吸动态. (未刊资料)

林中细根呼吸在 7 月上旬达最高峰 $\left(4.7 \mu \mathrm{mol} \mathrm{m} \mathrm{m}^{-3}\right.$ $\mathrm{s}^{-1}$ ) 后下降, 5月根呼吸对土壤呼吸的贡献为 $33 \%$ $\sim 62 \%$, 10 月则降低到 $12 \% \sim 16 \%$; 但细根呼吸末 出现明显的昼夜变化。而 Vose 和 Ryan(2002) 发现 白松 (Pinus strobus) 细根呼吸除在秋初较大外 (1.64 $\left.\mu \mathrm{mol} \mathrm{kg}^{-1} \cdot \mathrm{s}^{-1}\right)$,一年中细根呼吸几乎保持恒定

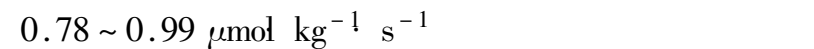

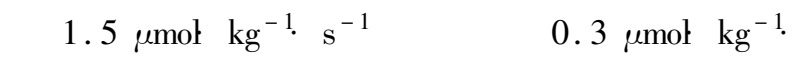
$\mathrm{s}^{-1}$ )。Ryan 等 (1997) 发现寒温带森林根呼吸早春最 大秋末最小。Edwards (1991) 发现在 3、5、8、12 月 时火炬松 (Pinus taeda) 根呼吸分别占土壤呼吸的 $67 \% 、 78 \% 、 54 \%$ 和 $67 \%$; 而 Lin 和 Ehleringer (1997) 则发现一年生黄杉 (Pseudotsuga) 根呼吸在 4、6、8 和 10 月时对土壤呼吸的贡献分别为 $28 \% 、 12 \% 、 25 \%$ 和 30\%。根呼吸因土壤深度而异，Pregitzer 等(1998) 发现表层土 $(0 \sim 10 \mathrm{~cm})$ 中糖械 ( Acer saccharum) 根 $(\Phi<0.5 \mathrm{~mm})$ 呼吸速率比深层的高 $40 \%$ 。Uchida 等 (1998) 发现黑云杉 ( Picea mariana) 根呼吸对土壤呼
吸的贡献在 L、 LF、A 和 $\mathrm{E}$ 层分别为 $6 \% 、 80 \% 、 43 \%$ 和 0 。Flanagan 和 $\operatorname{Van}(1977)$ 亦发现黑云杉的根呼吸 对土壤呼吸的贡献在 $\mathrm{L}$ 层和 $\mathrm{H}$ 层分别为 $80 \%$ 和 $90 \%$ 。

\section{2 林木根呼吸测定方法}

根呼吸的测定方法有直接和间接测定，直接测 定法有离体根法 (In vitro root methods)、PVC 管气室 法(Cuvette methods)、同位素法(Isotopic methods)等; 间接测定有排除根法 (Root exclusion methods) 等。用 排除根法测定的根呼吸包括根际微生物呼吸。

\section{1 间接测定方法一一排除根法 (Root exclusion} methods)

排除根法是通过测定有根和无根土壤呼吸间的 差异来间接估计根呼吸的, 该方法测定值含根呼吸 和根际微生物呼吸。1) 切除根法 (Root removal) : 将 小区内的所有根切除后移去，土壤尽量按原层次回 填, 并调节土壤容重与原土壤相对一致, 同时小区四 
周用障碍物隔离以阻止根向小区内生长。2)挖壕法 (Trenching):在小区四周挖壕沟切断根 (不移走) 后 插入障碍物以阻止根向小区内生长。3) 林隙分析 (Gap analysis) :将一定面积的地上植被除去以形成 林隙, 测定林隙与林内土壤呼吸的差异来间接估计 根呼吸。挖壕法要考虑如何排除小区内非正常死亡 根分解的影响, 这可通过挖壕沟后定期观测土壤呼 吸变化或周期性地取壕沟内的细根以判断非正常死 亡根分解基本完成的时间来解决 (Hanson et al. , $2000)$ 。林隙分析与挖壕法存在同样的问题, 但若加 以适当处理, 该方法还是比较可行的, 若林隙是由于 单棵树死亡或风倒形成的尤为如此。但林隙面积不 能太小以防周围植被的根长入测定区域, 且又不能 太大以防林隙和林内土壤微域环境差异太大。切除 根方法虽不存在非正常死亡根分解问题, 但该方法 对土壤的干扰较大而影响测定结果。

排除根法由于对土壤的干扰通常 导致土壤初始 呼吸速率增加, 但随后呼吸速率逐渐衰减, 并最终 (一般 3４月) 恢复到原来水平 (Bowden et al. , 1993 ; 同时该方法还存在切除根后造成土壤湿度增 加的问题; 壤干扰、根和土壤间相互作用消失、裸 土和有植被土壤间温度和湿度的差异都可能影响其 测定结果(Rochette et al .,1999)。但该方法相对简 单, 常用于森林生态系统中, 可对根呼吸和土壤呼吸 作出可靠估计。

Högberg 等 (2001) 近来提出了一种新的间接测 定根呼吸的方法一一环割实验 (Girdling experiment), 即将韧皮部环割后 (保留木质部使之继续运输水分) 观测土壤呼吸的变化来估计根呼吸, 但由于该方法 需要大量的重复工作而限制了其应用 (Högberg et al. 2001)。

\section{2 直接测定方法}

2.2.1 离体根法(Isolated/ in vitro root methods)

从林木根系中切除待测根后, 在大气或土壤 $\mathrm{CO}_{2}$ 浓度环境下迅速测定离体根呼吸( Zogg et al. , 1996 ;Widén \& Majdi，2001)。该方法主要用于便携 式气体分析仪不常用的早期, 操作相对简单, 且可以 测定对温度的响应曲线, 常用于森林生态系统中。 缺点一是破坏性较大, 且出现创伤呼吸(切断的根有 较高的呼吸速率)，但可通过离体后迅速测定和密封 根伤口的办法将其影响减至最小;二是不能重复测 定同一根样品; 三是还存在由少数几条根测定结果 推算整个生态系统根呼吸的尺度转换误差问题。另 外, 离体后开始根呼吸测定时间的不同亦会影响其
结果, 因为根离体后其呼吸速率随时间而迅速下降, 如 Rakonczay 等(1997b) 发现红花械 (Acer rubrum)、沼 地红栎( Quercus rubra)，北美乔松(Pinus strobes)细根 切除 1 分钟到 10 小时内根呼吸随时间明显下降，红 花械和北美乔松根切除 5 分钟后、沼地红柇切除 15 分钟后分别下降 $37 \% 、 52 \%$ 和 $33 \%$ 。

\subsection{2 同位素法(Isotopic methods)}

该方法消除了对土壤的干扰，可原位测定根呼 吸和微生物呼吸, 但其缺点主要是实验设备复杂增 加了测定难度和分析成本等。同位素法广义上可分 为:1) 间歇标记 (Pulse labeling) ,2) 重复间歇标记 (Repeated pulse labeling) 和 3) 连续标记 (Continuous labeling)。放射性 ${ }^{14} \mathrm{C}$ 或稳定 ${ }^{13} \mathrm{C}$ 都可作为示踪物，每 种方法给出的关于植物 $\mathrm{C}$ 分配和根呼吸对土壤呼 吸的贡献的信息却稍有差异(Hanson et al，,2000)。

间歇标记和重复间歇标记: 间歇标记是通过添 加 $\mathrm{C}$ 示踪物测定植物体内标记 $\mathrm{C}$ 的分布和特定时 间内植物地上和地下部分呼吸中标记 $\mathrm{C}$ 的丰度而 量化。它适合于生长在密闭气室内的小型植物同化 的 ${ }^{14} \mathrm{CO}_{2}$ 去向的追踪。由于植物中易分解的碳化合 物较易标记, 用此法可能高估了根呼吸。重复间歇 标记是间歇标记的变换形式之一, 即在不同生长季 节添加同位素标记的 $\mathrm{CO}_{2}$ 。尽管间歇标记或重复间 歇标记适用于野外条件且简单直观, 对研究植物体 内碳分配的动态 (Paterson et al . , 1997) 和定期测定 根呼吸是比较理想的, 但间歇标记和重复间歇标记 存在健康和安全问题, 在野外条件下应用尚受一定 的限制。

连续标记法 :连续标记是指在室内或野外条件 下，在植物不同生长季节里加入特殊标记 $\mathrm{C} 。$ 。连续 标记优于间歇标记之处是: 1) 它能提供较均匀标记 的植物碳库 2) 经常处于稳定状态且易于计算。缺 点是:1)不适合研究瞬时植物碳动态,2)所需设备昂 贵且笨重, 应用到野外很困难( 尤其是在森林生态系 统中）３）难以区分根呼吸和土壤有机质分解释放的 $\mathrm{CO}_{2}$ (Hanson et al . 2000)。实验室内则可用于连续 标记具示踪 ${ }^{14} \mathrm{C}$ 的小型植物, 但测定较大树木则不合 适。

1) 核弹- ${ }^{14} \mathrm{C}$ ( Bomb derived $\left.{ }^{14} \mathrm{C}\right): 20$ 世纪 50 年 代和 60 年代初的核武器实验增加了大气 $\mathrm{CO}_{2}$ 的 ${ }^{14} \mathrm{C}$ 含量 结果开创了使植物和土壤碳库的标记比短期 连续标记更均匀的全球长期标记试验。根呼吸对土 壤呼吸的贡献可通过测定大气 $\mathrm{CO}_{2}$ 、土壤有机质和 土壤呼吸测定中 ${ }^{14} \mathrm{C}$ 的丰度来量化。 
2) 稳定同位素法 (Stable isotope techniques) : 稳 定同位素法是在光合途径变化 (如生长在前茬是 $\mathrm{C}_{3}$ 植物的土壤上的 $\mathrm{C}_{4}$ 植物 ) 或大气 $\mathrm{CO}_{2}$ 中 ${ }^{13} \mathrm{C}$ 丰度长 期变化的基础上来量化各分室对土壤呼吸的贡献。 具 $\mathrm{C}_{3}\left(\delta^{13} \mathrm{C}\right.$ 为 $\left.12 \% 0\right)$ 和 $\mathrm{C}_{4}\left(\delta^{13} \mathrm{C}\right.$ 为 $26 \%$ ) 光合途径的 植物 C 同位素构成约相差 $14 \%$ 。该方法测定根对 土壤呼吸的贡献主要的局限是在没有光合途径改变 的情况下根呼吸产生的 $\mathrm{CO}_{2}$ 和土壤有机质分解产生 的 $\mathrm{CO}_{2}$ 间的同位素差异较小而无法有效区分。

3) FACE 实验 (FACE experiments): 在 FACE 中 应用 ${ }^{13} \mathrm{C}$ 标记使源于根的 $\mathrm{CO}_{2}$ 从土壤呼吸中区分出 来。连续标记法应用于 FACE 实验也有很大的局 限, 如当 ${ }^{13} \mathrm{C}$ 讯号进入其它有机碳库时亦无法区分源 于根呼吸和有机 $\mathrm{C}$ 库分解释放的 ${ }^{13} \mathrm{C}$ 标记, 且此法 还受植物对 FACE 响应的限制, 因为 $\mathrm{CO}_{2}$ 浓度升高 常导致根呼吸增加(Schlesinger \& Andrews ,1999)。

\subsubsection{PVC 管气室法(Cuvette methods)}

从树干基部出发沿根生长方向寻找合适的测定 根安装 PVC 管气室。粗根 PVC 管气室安装完后可 连续测定 细根 PVC 管气室则只能即测即安装 (因 细根周转快)。因细根较脆嫩和根际微生物等问题， 测定细根呼吸时有一定难度 (Rakonczay et al., 1997b ; Vose \& Ryan 2002)。在 20 世纪 70 80 年代 末此方法国外应用较多, 优点是可重复和连续测定 同一根样品, 缺点是只能测定表层的根; 安装 PVC 管气室时经常干扰立地, 改变了根微环境, 操作难度 大，工作量较大，重复性差; 且因经常发生对待测根 的机械干扰而有可能使根系发生栓化。Rakonczay 等(1997a)在此基础上设计了原位测定细根呼吸的 新式气体交换系统(图 1)。该系统中柔韧的 PVC 管 允许封闭的根和外界土壤进行气体、水分和养分的 交换，且防止了大型生物的进入。在一定程度上避 免了上述一些缺点，但对根系生理代谢活跃根毛的 处理仍有难度, 有待改进。如可通过供给 PVC 管模 拟的或实际土壤溶液使其更接近实际土壤条件, 通 过应用不同的营养液来估计与养分吸收相关的呼 吸。

由于不同研究间采用的根呼吸测定方法不同， 因而非常需要对不同测定方法的结果和精度进行比 较, 但目前此方面的报道极少, 以致限制了不同研究 之间的比较和降低了研究结果的可比性。不过上述 方法各有自己的优点可以吸取 :1) 排除根法和离体 根法相对简单, 成本低, 可用于与原位测定结果比 较, 是测定森林生态系统林木根呼吸的常用方法
(Rochette et al. 1999 ;Widén \& Majdi，2001)。2)稳 定同位素法在原位可将土壤呼吸分为根呼吸和土壤 有机质分解，对土壤-植物系统的干扰小，因而优于 排除根法和离体根法, 但高成本和 (或)缺少适当的 专业技术以及当在野外使用时如何定量等问题使该 方法应用受到一定限制。3) 核弹- ${ }^{14} \mathrm{C}$ 法 (Bomb- $\left.{ }^{14} \mathrm{C}\right)$ 可能是最好的区别现有森林生态系统中各种 $\mathrm{CO}_{2}$ 来 源对土壤呼吸贡献的方法, 但分析的难度和高成本 亦限制它成为一种常规方法。4) PVC 管气室法通过 改进亦不失为一种可行方法。不过每种方法在广泛 应用之前, 都应进一步检验其功效并与其它方法比 较。

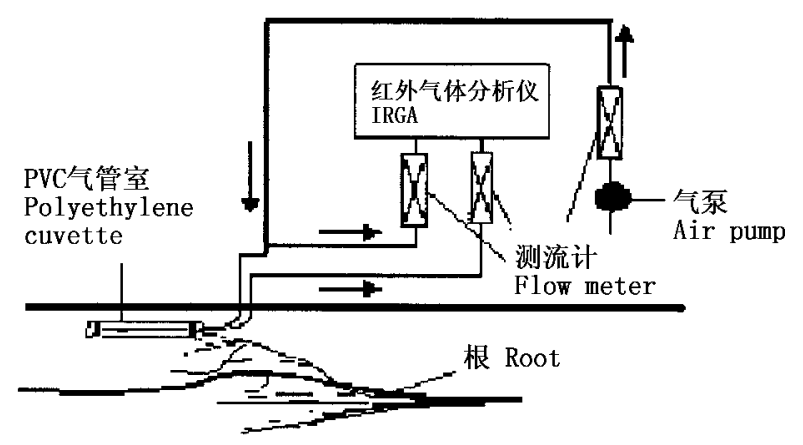

图 1 PVC 管气室法原位测定根呼吸示意图

Fig.1 Schematic diagram of polyethylene cuvette methods measuring root respiration (modified from Rakonczay et al. , 1997a)

\section{3 影响林木根呼吸的因素}

研究根呼吸的调控因素对构建根呼吸的动态模 型至关重要。影响林木根系呼吸的主要因素有土壤 温度、根直径大小、根组织 $\mathrm{N}$ 浓度、环境 $\mathrm{CO}_{2}$ 浓度、 养分有效性及土壤湿度等 (Widén \& Majdi，2001； Desrochers et al ., 2002 ; Högberg et al . 2002)。

\section{1 土壤温度与林木根呼吸}

根呼吸对温度有强烈的敏感性。多数研究者发 现林木细根呼吸随土壤温度的升高呈指数增加 (Burton et al. ,1996; Zogg et al., 1996 ; Bouma et al.,1997;Widén \& Majdi，2001)。Boone 等(1998)发 现 1985 年生温带混交林根/根际微生物呼吸对温度 的敏感性比全土和无根土壤的大 根/根际微生物呼 吸的 $Q_{10}$ 值是 4.6 , 而无根土壤和全土的则分别为 2.5和 3.5。Gansert (1994) 发现 10 年生山毛榉细根 呼吸与土壤温度呈高度相关, 并遵循阿列纽斯特征 曲线( Arrhenius type curve)。Desrochers 等(2002)亦发 现 55 年生白杨 (Populus tremuloides) 林细根呼吸速率 与土壤温度间呈非线性关系, $Q_{10}$ 在 $T=5 \sim 15{ }^{\circ} \mathrm{C}$ 时 
为 3.90 , 在 $T=15 \sim 25{ }^{\circ} \mathrm{C}$ 时为 2.19 。但 Burton \& Pregitzer (2003) 在糖械和赤松细根呼吸的野外测定 时没有发现其与温度相适应; Vose 和 Ryan(2002) 亦 发现土壤温度对白松根呼吸的季节动态影响较小， 并认为土壤温度高时细根呼吸速率高可能是对生长 的反应。理论上,土壤温度增加将导致根系呼吸增 加, 但是若出现根对温度变化适应的话, 那么实际的 $Q_{10}$ 将比预计的低，如对高温的适应可导致林木根呼 吸速率比预计的低, 而对低温的适应则可使根呼吸 速率比预计的高 (Burton et al.,1996)。

\section{2 根直径大小与根呼吸}

通常林木根呼吸速率随根直径的增大而降低, 细根的呼吸速率比粗根的高 (Elbert et al.,1992; Vose \& Ryan ,2002)。Rakonczay 等 (1997b) 研究发现 樱桃 (Prunus serotina)、红花械和沼地红栎的细根 $(\Phi$ $<2.0 \mathrm{~mm}$ ) 呼吸速率比粗根的高 6 倍多;Pregitzer 等 (1998) 发现糖槭林细根 $(\Phi<0.5 \mathrm{~mm})$ 呼吸速率比粗 根的大 2.4 3.4 倍; Desrochers 等 (2002) 发现 55 年 生加拿大白杨 (Populus basamifera) 林在 $T=15{ }^{\circ} \mathrm{C}$ 时 细根平均呼吸速率生长季期内为 $1290 \mu \mathrm{mol} \mathrm{m} \mathrm{m}^{-3}$. $\mathrm{s}^{-1}$, 休眠期内为 $660 \mu \mathrm{mol} \mathrm{m}^{-3} \cdot \mathrm{s}^{-1}$, 而粗根的呼吸 速率在晚秋休眠期仅为 $370 \mu \mathrm{mol} \mathrm{m}^{-3} \cdot \mathrm{s}^{-1}$, 发芽期 和夏初仅为 $200 \mu \mathrm{mol} \cdot \mathrm{m}^{-3} \cdot \mathrm{s}^{-1}$; Widén 和 Majdi (2001) 发现松树-云杉混交林 5 月根呼吸对土壤呼 吸的贡献为 $62 \%$, 其中有 $58 \%$ 来自直径 $<5 \mathrm{~mm}$ 的 根呼吸。Bhaskar 等 (1993) 等发现根代谢活性高的 根毛是专门的呼吸器官, 根尖呼吸速率比根其它部 分的高 细根呼吸速率比粗根的高, 这可能与根中分 生组织和非分生组织所占比例不同所致。一般来说 粗根仅占总根系呼吸的 $30 \%$ 左右, 且粗根呼吸对温 度的敏感性比细根的低。具外生菌根的根呼吸速率 亦比无菌根的高 $6 \% \sim 40 \%$, 有的树种甚至高达 300\% (Rakonczay et al . , 1997b)。

\section{3 根 $\mathrm{N}$ 浓度与根呼吸}

一般来说根呼吸速率随根组织 $\mathrm{N}$ 浓度的升高 而增加 (Amthor,2000 ; Ryan et al. ,1996)。Zogg 等 (1996) 发现北方阔叶林中细根呼吸随根含 $\mathrm{N}$ 量增加 而呈线性增加; Desrochers 等 (2002) 发现根呼吸速率 与根组织 $\mathrm{N}$ 浓度呈较好的正相关; Widén 和 Majdi (2001) 也发现松树-云杉混交林内根呼吸随根 N 浓 度升高而升高 (相关性显然达显著水平), 但不如根 呼吸与土壤温度间的相关性好; Pregitzer 等 (1998) 发 现糖械林根 $\mathrm{N}$ 浓度可解释不同立地、根径级大小和 深度间根呼吸变化的 $70 \%$;Burton 等 (1996) 在 4 种
硬阔叶林发现细根呼吸和细根 $\mathrm{N}$ 浓度显著相关; Burton 等(2002) 发现裸子植物比被子植物有较低的 根呼吸速率主要是因为裸子植物细根 $\mathrm{N}$ 浓度较低, 且较温暖立地的根 $N$ 浓度和根呼吸速率 (相同温度 下)比较寒冷立地的低。Vose 和 Ryan (2002) 发现细 根生长呼吸与根 $\mathrm{N}$ 浓度无关, 但粗根生长呼吸则与 根 $\mathrm{N}$ 浓度有关 粗根和细根的维持呼吸则均与根组 织 $\mathrm{N}$ 浓度有良好的相关性;Ryan (1991) 和 Ryan 等 (1996)亦表明根维持呼吸与根组织 $\mathrm{N}$ 呈良好正相 关。这主要是因为根维持呼吸为根内蛋白质的修复 和更新提供能量, 室内研究表明 $85 \%$ 的维持呼吸与 蛋白质的周转有关 (Bouma et al , 1994 ;Ryan et al. , 1996)。

\section{4 环境 $\mathrm{CO}_{2}$ 浓度与根呼吸}

环境 $\mathrm{CO}_{2}$ 浓度主要通过两种途径对根呼吸速率 产生影响 通过改变测定时根区 $\mathrm{CO}_{2}$ 浓度梯度而直 接影响根呼吸速率; 通过大气 $\mathrm{CO}_{2}$ 浓度改变而改变 植物生长速率或植物营养体化学组成，从而间接影 响根呼吸 (Hamilton et al.,2002; Burton et al. , 2002)。

Qi 等(1994)发现 350 ppm CO 2 浓度下测定的花 旗松 ( Pseudotsuga menzjessi) 细根生长呼吸和维持呼 吸分别是在 $1000 \mathrm{ppm}$ 下测定值的 2.0 倍和 2.5 倍。 Burton 等(1997) 则发现 $350 \mathrm{ppm}$ 和 $1000 \mathrm{ppm} \mathrm{CO}$ 浓 度下测定的糖械离体细根呼吸速率相差 1.2 倍。 Cliton 和 Vose (1999) 发现在大气 $\mathrm{CO}_{2}$ 浓度 $(345 \sim 397$ $\mathrm{ppm}$ ) 和土壤 $\mathrm{CO}_{2}$ 浓度 (345 1 $122 \mathrm{ppm}$ )下测定的成 年北美乔松根呼吸在 5、6 和 12 月差异不显著, 而其 余月份测定结果差异均显著, 以 9 月的差异最大 (大 气 $\mathrm{CO}_{2}$ 浓度下测定值是土壤 $\mathrm{CO}_{2}$ 浓度下的 36 倍)； 而大气环境下测定的全年平均细根呼吸强度则是土 壤环境下的 2.5 倍, 且在土壤环境下测定的细根呼 吸强度与土壤环境中 $\mathrm{CO}_{2}$ 浓度呈线性负相关。但 Bouma 等 (1997) 却发现柑橘 (Citrus volkameriana) 细 根呼吸并无上述响应。Burton 等(2002) 亦发现加拿 大白杨、白云杉 (Picea glauca) 、糖械、脂松 (Pinus resinosa)、湿地松 ( $P$. elliottii ) 等 9 个树种离体细根 在 $350 \mathrm{ppm}$ 和 $1000 \mathrm{ppm}$ 下的呼吸作用十分相近, 即 $R_{350} / R_{1000}$ 接近 1.0(0.97 1.07); 同时他们认为原 位细根呼吸速率测定不受 $\mathrm{CO}_{2}$ 浓度影响。McDowell 等(1999)发现异叶铁杉 ( Tsuga heterophylla) 根维持呼 吸明显受土壤 $\mathrm{CO}_{2}$ 浓度影响, 但生长呼吸却未受影 响; 且细根和粗根对土壤 $\mathrm{CO}_{2}$ 浓度的响应不同。 Hamilton 等 (2002) 在北卡来罗纳州火炬松为优势种 
和美国枫香 (Liquidambar styraciflua)、北美我鸟掌楸 (Liriodendron tulipifera) 为亚优势种的杜克森林 FACE 实验中发现, $\mathrm{CO}_{2}$ 浓度提高 1 倍后, 与对照相比粗根 呼吸略有升高 (从 $61 \mathrm{G} \mathrm{m}^{-2} \mathrm{a}^{-1}$ 升至 $64 \mathrm{Ci} \mathrm{m}^{-2}$. $\mathrm{a}^{-1}$ ), 但细根呼吸却有明显下降 (从 $662 \mathrm{C} \mathrm{m}^{-2} \mathrm{a}^{-1}$ 降至 $555 \mathrm{C} \mathrm{m}^{-2} \mathrm{a}^{-1}$ ) (Hamilton et al .,2002)。

\section{5 土壤养分有效性与根呼吸}

养分有效性增加 (施肥) 通常可促进根呼吸 (Ryan et al. ,1996; Zogg et al., 1996 ; Pregitzer et $a l .2000)$ 。Borken 等 (2002) 发现施有机肥使 90 年 生挪威云杉 ( Picea abies) 林根呼吸明显增加。但 Maier 和 Kress (2000) 则发现施肥对 11 年生火炬松根 呼吸无影响, 因为施肥虽然降低了细根生物量, 但增 加了粗根生物量, 且施肥提高了粗根的 $\mathrm{N}$ 浓度, 因 而虽然细根维持呼吸和生长呼吸降低了, 但由于粗 根生物量增大和比根呼吸速率增加而使粗根呼吸增 加，所以总的根呼吸保持不变; 但施肥使 $0 \sim 15 \mathrm{~cm}$ 土层根呼吸占总根呼吸比例下降 (细根生物量降 低), 而使底层 $(15 \sim 20 \mathrm{~cm}$ ) 的根呼吸增加 (粗根生物 量增大)。

\section{6 土壤湿度与根呼吸}

土壤湿度对林木根呼吸的影响比较复杂 (Lambers et al ., 1996 ; Gansert , 1994 ; Burton \& Pregitzer , 2003)。Lambers 等(1996) 发现在植物受水分胁迫时 降水量增加可使比根呼吸速率 (Specific root respiration rate) 增加, 而降雨量减少则降低了比根呼吸。 Burton 和 Pregitzer (2003) 发现土壤温度和土壤水分 有效性共同解释了赤松 (Pinus resinosa) 和糖械林人 工林内根呼吸速率变化的 $76 \%$ 和 $71 \%$ 。 Gansert (1994) 则发现林木细根呼吸速率与枯枝落叶层含水 量呈负相关, 而Widén 和 Mgidi (2001) 却认为细根呼 吸与土壤含水量无关。

\section{4 展 望}

1) 鉴于根呼吸测定目前缺乏成熟的方法, 今后 应在对林木根呼吸 (生长呼吸、维持呼吸) 不同测定 方法分析比较的基础上, 确定不同条件下测定根呼 吸最合适的方法, 并寻求更通用、简便和精确的测定 根呼吸方法。同时, 应解决不同测定方法结果间的 可比性和数据互接共享问题, 以利于在更大的尺度 上分析森林生态系统根呼吸动态和调控机理。

2) 为科学评价各种森林变化、营林措施和环境 因素对森林土壤的净 $\mathrm{C}$ 交换 (源汇功能)的影响,精 确估计根系呼吸对土壤呼吸的贡献显得极为重要，
因而探讨有效分离根呼吸和根际微生物呼吸的方法 则是亟待解决的问题。

3) 目前有关林木根呼吸动态的研究时间普遍 偏短，鉴于各种生态因子作用的复杂性，今后应对森 林生态系统根呼吸动态进行长期的定位研究, 以尽 量消除根呼吸动态中的不确定性, 为森林生态系统 C 循环模型构建提供坚实的基础数据。

4) 应进一步扩大对不同气候带、不同森林类 型、不同立地条件、不同生长发育阶段、不同土壤深 度、不同功能及不同营林措施下森林生态系统根呼 吸动态及调控机理研究。应加强国际协作, 在全球 $\mathrm{CO}_{2}$ 通量网建设中使用标准方法进行根呼吸的定位 观测, 使研究尺度从生态系统扩大到区域或全球水 平, 为区域及全球的 $\mathrm{C}$ 预算和 $\mathrm{C}$ 循环模型的构建提 供科学依据。同时, 应加强分子水平的研究, 以探讨 遗传特性、基因型等树种内在特性对根呼吸的影响。

5) 加强林木根呼吸对 $\mathrm{CO}_{2}$ 浓度升高、温度升 高、酸沉降、 $\mathrm{N}$ 沉降和 $\mathrm{U}-\mathrm{VB}$ 辐射增加等全球变化响 应及机制的研究。

6) 随着植物生理学、森林生态学、生物化学、生 物数学和分子生物学不断取得突破, 这些学科对根 呼吸的合作研究必将为森林生态系统根呼吸研究做 出新的贡献。

\section{参 考 文 献}

Amthor, J. S. 2000. The McCree-de wit-penning de vries-thornley respiration paradigms: 30 years later. Annals of Botany, 86: $1 \sim$ 20 .

Andrews, J. A., K. G. Harrison \& W. H. Schlesinger. 1997. Separation of root from soil respiration in the field using stable isotope tracers. Agronomy Journal-Abstracts, 209.

Andrews, J. A., K. G. Harrison, R. Matamala \& W. H. Schlesinger. 1999. Separation of root respiration from total soil respiration using carbon-13 labeling during Free-Air Carbon dioxide Enrichment (FACE) . American Journal of Soil Science Society, 63: $1429 \sim 1435$.

Bhaskar, V., G. P. Berlyn \& J. H. Connolly. 1993. Root hairs as specialized respiratory cells: a new hypothesis. Journal of Sustainable Forestry, 1: $107 \sim 125$.

Bonan, G. B. 1993. Physiological controls of the carbon balance of boreal forest ecosystems. Canadian Journal of Forest Research, 23: $1453 \sim 1471$.

Boone, R.D., K. J. Nadelhoffer, J. D. Canary \& J. P. Kaye. 1998. Roots exert a strong influence on the temperature sensitivity of soil respiration. Nature, 396: $570 \sim 572$.

Borken, W. , A. Muhs \& F. Beese. 2002. Application of compost in spruce forests: effects on soil respiration, basal respiration and microbial biomass. Forest Ecology and Management, 159: 49 58.

Bouma, T. J. \& D. R. Bryla. 2000. On the assessment of root and soil respiration for soils of different textures: interactions with 
soil moisture contents and soil $\mathrm{CO}_{2}$ concentrations. Plant and Soil, 227: $215 \sim 221$.

Bouma, T. J., L. N. Kai, M. E. David \& P. L. Jonathan. 1997. Estimating respiration of roots in soil: interactions with soil $\mathrm{CO}_{2}$, soil temperature and soil water content. Plant and Soil, 195: $221 \sim 232$.

Bouma, T. J., D. R. Visser \& J. H. Janssen. 1994. Respiratory energy requirements and rate of protein turnover in vivo determined by the use of an inhibitor of protein synthesis and a probe to assess its effect. Physiologia Plantarum, 92:585 594 .

Bowden, R. D., K. J. Nadelhoffer, R. D. Boone, J. M. Melillo \& J. B. Garrison. 1993. Contribution of aboveground litter, belowground litter, and root respiration total soil respiration in a temperate mixed hardwood forest. Canadian Journal of Forest Research, 23: $1402 \sim 1407$.

Brumme, R. 1995. Mechanisms of carbon and nutrient release and retention in beech forest gaps. Plant and Soil, 168/169: 593 600 .

Burton, A. J. \& K. S. Pregitzer. 2003. Field measurements of root respiration indicate little to no seasonal temperature acclimation for sugar maple and red pine. Tree Physiology, 23: 273 280 .

Burton, A. J., K. S. Pregitzer \& R. W. Rues. 2002. Root respiration in North American forests: effects of nitrogen concentration and temperature across biomes. Oecologia, 131: $559 \sim 568$.

Burton, A. J., K. S. Pregitzer, G. P. Zogg \& D. R. Zak. 1996. Latitudinal variation in sugar maple fine root respiration. Canadian Journal of Forest Research, 26: $1761 \sim 1768$.

Burton, A. J., G. P. Zogg, K. S. Pregitzer \& D. R. Za. 1997. Effect of measurement $\mathrm{CO}_{2}$ concentration on sugar maple root respiration. Tree Physiology, 17: $421 \sim 428$.

Catricala, C.E., K. M. Newkirk, P. A. Steudler \& J. M. Melillo. 1997. Effect of soil warming onmicrobial and root respiration. Agronomy Journal-Abstracts, 284.

Casals, P. , J. Romanyà, J. Cortina, P. Bottner, M. Coûteaux \& V. R. Vallejo. 2000. $\mathrm{CO}_{2}$ efflux from a Mediterranean semi-arid forest soil. I. Seasonality and effects of stoniness. Biogeochemistry, 48: $261 \sim 281$.

Clinton, B.D. \& J. M. Vose. 1999. Fine root respiration in mature eastern white pine (Pinus strobus) in situ: the importance of $\mathrm{CO}_{2}$ in controlled environments. Tree Physiology, 19(7): $475 \sim 479$.

Coleman, D. C., M. D. Hunter, J. Hutton, S. Pomeroy \& S. J. Lloyd. 2002. Soil respiration from four aggrading forested watersheds measured over a quarter century. Forest Ecology and Management, 157:247 253.

Desrochers, A., S. M. Landhäusser \& V. J. Lieffers. 2002. Coarse and fine root respiration in aspen (Populus tremuloides). Tree Physiology, 22: $725 \sim 732$.

Dörr, H. \& K. O. Münnich. 1987. Annual variation in soil respiration in selected areas of the temperate zone. Tellus, 39B: 114 $\sim 121$.

Ebert, G. \& F. Lenz. 1991. Annual course of root respiration of apple trees and its contribution to the $\mathrm{CO}_{2}$ balance. Gartenbauwissenschaft, 56: $130 \sim 133$.

Edwards, N. T. 1991. Root and soil respiration responses to ozone in Pinus taeda L. seedlings. New Phytologist, 118: $315 \sim 321$.

Elbert, G. 1992. The root respiration of apple trees. II. Influence of endogenous factors. Erwerbsobstbau, 34(6) : $165 \sim 168$.

Ellsworth, D. C. 1999. $\mathrm{CO}_{2}$ enrichment in a maturing pine forest: are $\mathrm{CO}_{2}$ exchange and water status in canopy affected? Ecology, 80: $1955 \sim 1969$.
Epron, D., L. Farque, E. Lucot \& P. M. Badot. 1999. Soil $\mathrm{CO}_{2}$ efflux in a beech forest: the contribution of root respiration. Annals of Forest Science, 56: $289 \sim 295$.

Ewel, K.C., W.P. Jr. Cropper \& H. L. Gholz. 1987. Soil $\mathrm{CO}_{2}$ evolution in Florida slash pine plantations. II. Importance of root respiration. Canadian Journal of Forest Research, 17: 330 333.

Flanagan, P.W . \& C. K. Van. 1977. Microbial biomass, respiration and nutrient cycling in a black spruce taiga ecosystem. Ecological Bulletin, 25: $261 \sim 273$.

Gansert, D. 1994. Root respiration and its importance for the carbon balance of beech saplings (Fagus sylvatica L. ) in a montane beech forest. Plant and Soil, 167: 109 119 .

Hamilton, J. G., E. H. Delucia, K. George, S. L. Naidu, A. C. Finzi \& W. H. Schlesinger. 2002. Forest carbon balance under elevated $\mathrm{CO}_{2}$. Oecologia, 131: $250 \sim 260$.

Hanson, P. J., N. T. Edwards, C. T. Garten, J. A. Andrews, L. E. Rustad, T. G. Huntingdon \& R. D. Boone. 2000. Special Issue. Controls on soil respiration: implications for climate change: separating root and microbial contributions to soil respiration: a review of methods and observations. Biogeochemistry, 48: $115 \sim 146$.

Haynes, B. E. \& S. T. Gower. 1995. Belowground carbon allocation in unfertilized and fertilized red pine plantations in northern Wisconsin. Tree Physiology, 15: $317 \sim 325$.

Hogg, E. H. 1999. Simulation of interannual responses of trembling aspen stands to climatic variation and insect defoliation in western Canada. Ecological Modelling, 114: $175 \sim 193$.

Högberg , P. , A. Nordgren \& N. Buchmann. 2001. Large-scale forest girdling shows that current photosynthesis drives soil respiration. Nature, 411:789 792 .

Högberg, P. , A. Nordgren \& G. I. Ågren. 2002. Carbon allocation between tree root growth and root respiration in boreal pine forest. Oecologia, 132: $579 \sim 581$.

Irvine, J. \& B. E. Law. 2002. Contrasting soil respiration in young and old-growth ponderosa pine forests. Globle Change Biology, 8: $1183 \sim 1193$.

Kelting, D. L. , J. A. Burger \& G. S. Edwards. 1998. Estimating root respiration microbial respiration in the rhizosphere, and root-free soil respiration in forest soils. Soil Biology \& Biochemistry, 30: $961 \sim 968$.

Lambers, H. , I. Stulen \& A. Werf. 1996. Carbon use in root respiration as affected by elevated atmospheric $\mathrm{CO}_{2}$. Plant and Soil, 187: $251 \sim 263$.

Lin, G. H. \& J. R. Ehleringer. 1997. Carbon isotopic fractionation does not occur during dark respiration in $\mathrm{C}_{3}$ and $\mathrm{C}_{4}$. Plant Physiology, 114: $391 \sim 394$.

Maier, C. A. \& L. W. Kress. 2000. Soil $\mathrm{CO}_{2}$ evolution and root respiration in 11 year-old loblolly pine (Pinus taeda) plantations as affected by moisture and nutrient availability. Canadian Journal of Forest Research, 30: $347 \sim 359$.

McDowell, N. G., J. D. Marshall, J. Qi \& K. Mattsson. 1999. Direct inhibition of maintenance respiration in western hemlock roots exposed to ambient soil carbon dioxide concerntrations. Tree Physiology, 19: 599 605.

Morén, A.S. \& A. Lindroth. 2000. $\mathrm{CO}_{2}$ exchange at the floor of a boreal forest. Agriculture Forest Meteorology, 101: 1 14 .

Nakane, K., T. Kohno \& T. Horikoshi. 1996. Root respiration before and just after clear-felling in a mature deciduous, broadleaved forest. Ecological Research, 11: $111 \sim 119$.

Ohashi, M., K. Gyokusen \& A. Saito. 2000. Contribution of root 
respiration to total soil respiration in a Japanese cedar ( Cryptomeria japonica D. Don) artificial forest. Ecological Research, 15: $323 \sim 333$.

Paterson, E., J. M. Hall, E. A. S. Rattray, B. S. Grifiths, K. Ritz \& K. Killham. 1997. Effect of elevated $\mathrm{CO}_{2}$ on rhizosphere carbon flow and soil microbial processes. Global Change Biology, 3: $363 \sim 377$.

Pregitzer, K. S., D. R. Zak, J. Maziasz, J. DeForest, P. S. Curtis \& J. Lussenof. 2000. Interactive effects of atmospheric $\mathrm{CO}_{2}$ and soil- $\mathrm{N}$ availability on fine roots of Populus tremuloides. Ecological Applications, 10: $18 \sim 33$.

Pregitzer, K. S., M. J. Laskowski, A. J. Burton, V. C. Lessard \& D. R. Zak. 1998. Variation in sugar maple root respiration with root diameter and soil depth. Tree Physiology, 18: 665 670 .

Raich, J. W. \& W. H. Schlesinger. 1992. The global dioxide flux in soil respiration and its relationship to vegetation and climate. Tellus, 44B: $81 \sim 99$.

Raich, J. W. \& A. Tufekcioglu. 2000. Vegetation and soil respiration: correlations and controls. Biogeochemistry, 48: $71 \sim 90$.

Rakonczay, Z., J. R. Seiler \& L. J. Samuelson. 1997a. A method for the in situ measurement of fine root gas exchange of forest trees. Environmental and Experimental Botany, 37:107 113.

Rakonczay, Z., J. R. Seiler \& D. L. Kelting. 1997b. Carbon efflux rates of fine root of three tree species decline shortly after excision. Experimental Botany, 38: $243 \sim 249$.

Rey, A., E. Pegoraro, V. Tedeschi, I. D. Parri, P. G. Jarvis \& R. Valentini. 2002. Annual variation in soil respiration and its components in a coppice oak forest in Central Italy. Global Change Biology, 8: $851 \sim 866$.

Rochette, P., L. B. Flanagan \& E. G. Gregorich. 1999. Separating soil respiration into plant and soil components using analyses of the natural abundance of carbon-13. Soil Science Society of America Journal, 63: $1207 \sim 1213$.

Ryan, M.G. 1991. Effects of climate change on plant respiration. Ecological Applications, 1:157 167 .

Ryan, M. G., R. M. Hubbard, S. Pongracic, R. J. Raison \& R. E. McMurtrie. 1996. Foliage, fine-root, woody-tissue and stand respiration in Pinus radiata in relation to nitrogen status. Tree Physiology, 16: $333 \sim 343$.

Ryan, M. G., M. G. Lavigne \& S. T. Gower. 1997. Annual carbon cost of autotrophic respiration in boreal forest ecosystems in relation to species and climate. Journal of Geophysical Research, 102: $871 \sim 883$.

Schlesinger, W.H. \& J. A. Andrews. 1999. Soil respiration and the global carbon cycle. Biogeochemistry, 48: $7 \sim 20$.

Shangguan Z.P. ( 上官周平), M. A. Shao(邵明安) \& J. Dycknans. 2000. The loss of carbon with respiration of beech roots in soil. Acta Pedologica Sinica (土壤学报), 37:549 552. (in Chinese with English abstract)

Stoyan, H., H. De-Polli, S. Böhm, G. P. Robertson \& A. P. Eldor. 2000. Spatial heterogeneity of soil respiration and related properties at the plant scale. Plant and Soil, 222: $203 \sim 214$.

Striegl, R. G. \& K. P. Wickland. 1998. Effects of a clear-cut harvest on soil respiration in a jack pine - lichen woodland. Canadian Journal of Forest Research, 28: $534 \sim 539$.

Thierron, V. \& H. Laudelout. 1996. Contribution of root respiration to total $\mathrm{CO}_{2}$ efflux from the soil of a deciduous forest. Canadian Journal of Forest Research, 26: $1142 \sim 1148$.

Toland, D. E. \& D. R. Zak. 1994. Seasonal patterns of soil respiration in intact and clear-cut northern hardwood forests. Canadian Journal of Forest Research, 24: $1711 \sim 1716$.

Uchida, M., T. Nakatsubo, T. Horikoshi \& K. Nakane. 1998. Contribution of micro-organisms to the carbon dynamics in black spruce (Picea mariana) forest soil in Canada. Ecological Research, 13: $17 \sim 26$.

Vose, J. M. \& M. G. Ryan. 2002. Seasonal respiration of foliage, fine roots, and woody tissues in relation to growth, tissue $\mathrm{N}$, and photosynthesis. Global Change Biology, 8:164 175 .

Widén, B. \& H. Majdi. 2001. Soil $\mathrm{CO}_{2}$ efflux and root respiration at three sites in a mixed pine and spruce forest: seasonal and diurnal variation. Canadian Journal of Forest Research, 31:786 796.

Zogg, G. P., D. R. Zak, A. J. Burton \& K. S. Pregitzer. 1996. Fine root respiration in northern hardwood forests in relation to temperature and nitrogen availability. Tree Physiology, 16: $719 \sim 725$. 